\title{
Efecto de las características físicoquímicas y biológicas del suelo en el crecimiento de Pinus tecunumanii en Satipo
}

\author{
Effect of the physical, chemical and biological \\ characteristics of the soil on the growth of Pinus tecunumanii in Satipo
}

\author{
aLlallico Manzanedo, F; Marcelo Oyague, C. \\ Facultad de Ciencias Agrarias - Satipo / Universidad Nacional del Centro del Perú \\ Email: fllallico@uncp.edu.pe
}

\section{Resumen}

En el presente estudio, las plantaciones con cinco años de Pinus tecunumanii de la Estación Experimental Agrícola Satipo, ubicadas en la provincia de Satipo, presentaron plantas con diferentes tasas de crecimiento; por lo que, se evaluaron el crecimiento de las plantas y suelos en cada uno de los estratos, para luego relacionar las características del suelo y el crecimiento de las plantas, describiendo y correlacionando las variables en estudio, en calicatas y plantaciones. Los resultados muestran que la parcela de Pinus tecunumanii presenta tres estratos de crecimiento diferenciados, de los cuales el estrato alto, tiene el mayor crecimiento, tanto en altura de plantas como en diámetro de fuste, el estrato con menor crecimiento se encuentra en la parte superior de la parcela.

El suelo de los horizontes A, del estrato de mayor crecimiento, tiene mayor contenido de materia orgánica, mayor infiltración y profundidad efectiva; además, de mayor cantidad de lombrices, que los suelos del estrato de bajo crecimiento. Los suelos en cada uno de los estratos presentan bajo contenido de fósforo, potasio, calcio y magnesio, por lo que, se considera suelos de baja fertilidad, aunque el contenido de materia orgánica tiene un nivel medio. Además, no presentan problemas de acidez en el horizonte. Existe relación significativa entre el contenido de materia orgánica, la profundidad efectiva, la velocidad de infiltración, el contenido de lombrices y el crecimiento de las plantas, en general al incrementarse estas variables, se incrementa el crecimiento de Pinus tecunumanii.

Palabras clave: características de los suelos, fertilidad de suelos, crecimiento de plantas, materia orgánica e inorgánica, características fenotípicas de las plantas

\begin{abstract}
In the present study, the five-year Pinus tecunumanii plantations of the Satipo Agricultural Experimental Station, located in the province of Satipo, presented plants with different growth rates, hence the growth of the plants and soils of each one of the strata, were evaluated, and then relate the characteristics of the soil and the growth of plants, describing and correlating the variables under study in pits and plantations. The results show that the Pinus tecunumanii plot has three differentiated growth strata, of which the high stratum has the highest growth in both plant height and stem diameter, the stratum with the lowest growth is at the top of the plot.

The A horizons soil, from the stratum of greater growth, has a higher organic matter content, greater infiltration and effective depth, in addition to greater number of earthworms than the low growth stratum soils. The soils in each of the strata have a low content of phosphorus, potassium, calcium and magnesium, which is why they are considered low fertility soils, although the organic matter content has a medium level. It also has no acidity problems on the horizon. There is a significant relationship between the content of organic matter, the effective depth, the infiltration rate, the earthworms content and the plants growth, in general, as these variables increase, so does the growth of Pinus tecunumanii.
\end{abstract}

Keywords: soil characteristics, soil fertility, plant growth, organic and inorganic matter, phenotypic characteristics of plants 


\section{Introducción}

Las plantaciones forestales suministran productos importantes para su aprovechamiento, así como materia orgánica, que enriquecen al suelo con el aporte de nutrientes, mejorando su capacidad productiva; sin embargo, el desarrollo de estas plantaciones está influenciada por las características físicoquímicas y biológicas del suelo. Para que las plantaciones forestales prosperen, se debe realizar un manejo de la fertilidad del suelo, siendo importante destacar que para ello no solo los aspectos químicos, sino también, las propiedades físicas y biológicas del suelo donde se establecen las plantaciones forestales, los cuales varían según el clima y según el manejo que se le de al suelo (Arias, 2007).

El objetivo general fue evaluar el efecto de las características físicoquímicas y biológicas del suelo en el desarrollo de las plantaciones de Pinus tecunumanii en Satipo. Los objetivos específicos fueron: Determinar las características físicoquímicas y biológicas del suelo en las plantaciones de Pinus tecunumani; evaluar las características fenotípicas de las plantas de Pinus tecunumanii y; determinar la relación entre las características físicoquímicas y biológicas del suelo y las características fenotípicas de las plantas de Pinus tecunumanii.

Estudios realizados por Domínguez et al. (2017), indican que existe diferencia significativa de las variables de altura y diámetro entre los sitios y bloques, los mayores valores e incrementos se registraron en árboles de los sitios inferiores del terreno, suelos con mayor fertilidad. La altura promedio de los árboles fue $9.4 \mathrm{~m}$, con diámetros de $14.4 \mathrm{~cm}$ y volumen $60.2 \mathrm{~m}^{3} / \mathrm{ha}$; en cuanto a la profundidad, se observó que en la capa superficial $(0 \mathrm{~cm}$ - $5 \mathrm{~cm}$ ) presentan mayores contenidos y a mayor profundidad $(11 \mathrm{~cm}-15 \mathrm{~cm})$ estos decrecen, debido que en la capa superficial de los sitios y del testigo, existe acumulación de restos orgánicos, que al descomponerse liberan nutrimentos que incrementan su contenido y disponibilidad para las plantas, logrando un mayor crecimiento.

Martínez et al. (2013) realizaron el estudio sobre la "Influencia de variables físicas en la productividad de Pinus arizonica y Pinus engelmannii en el sur de Chihuahua, México" (p.35), con el objetivo de "caracterizar la influencia de variables físicas de los factores de clima, suelo y relieve en los bosques del sur de Chihuahua, México, clasificaron sitios con diferente productividad (alta, media y baja), usando modelos de índice de sitio" (p.37), donde concluyeron que "El uso de modelos de índice de sitio permitió definir niveles de productividad en la zona de distribución de Pinus arizonica y $P$. engelmannii; así como, identificar las variables con mayor relación sobre la productividad de los sitios en la región sur del estado de Chihuahua” ( p.45); como también, el "análisis de discriminante canónico y de varianza identificaron diferencias significativas en variables físicas como profundidad de suelo, la pendiente, disección vertical, temperatura mínima y máxima”(p.45). Mientras que en lo que se refiere a pedregosidad, porcentaje de suelo desnudo, grosor de mantillo y grosor de hojarasca, no mostraron diferencias significativas entre los estratos con diferente productividad.

Los arboles maderables, al igual que otros tipos de plantas, requieren de los mismos nutrientes, "unos en cantidades grandes llamados macronutrientes, tales como nitrógeno, fósforo, potasio, calcio, magnesio y azufre y otros que se requieren en cantidades pequeñas, por lo que se conoce como micronutrientes o elementos traza, entre ellos el hierro, cobre, cloro, manganeso, boro, zinc, níquel y molibdeno", siendo absorbidos estos por la planta en medio líquido (agua). Mientras que el carbono, hidrógeno y oxígeno absorbidos del aire vía fotosíntesis y a través del agua contenido en el suelo, (Alvarado, 2007, p. 17). Así mismo, "Un suelo de buena calidad no debe presentar compactación, debe ser de baja densidad aparente y resistencia a la penetración. La ausencia de compactación genera una adecuada y óptima porosidad que facilita la aireación, el drenaje y el almacenamiento de agua". (Vallejo y Quintero, 2013, p. 89).

Según (Rodríguez, 1982; Stoler y Relyea, 2011), citados por Martínez et al. (2013) “Las propiedades físicas que influyen en el crecimiento de algunas coníferas son $\mathrm{pH}$, material muerto y contenido de limo en la capa de $50 \mathrm{~cm}$ de suelo" (p.36). Al respecto, Cárdenas (2013) menciona que el Pinus tecunumanii es una especie que no tolera suelos muy húmedos, requiriendo suelos con un $\mathrm{pH}$ ligeramente ácido. Y, Grubb (1995) manifiesta que la fertilidad y las propiedades físicas, químicas y biológicas del suelo tienen un efecto directo sobre el crecimiento de las especies maderables.

En relación a la materia orgánica, Martínez et al. (2008), manifiestan que "el carbono orgánico del suelo (COS) se relaciona con la sustentabilidad de los sistemas agrícolas afectando las propiedades del suelo relacionadas con el rendimiento sostenido de los cultivos. El COS se vincula con la cantidad y disponibilidad de nutrientes del suelo, al aportar elementos como el $\mathrm{N}$ cuyo aporte mineral es normalmente deficitario. Además, al modificar la acidez y la alcalinidad hacia valores cercanos a la neutralidad, el COS aumenta la solubilidad de varios nutrientes”.

También, afirman que las lombrices cumplen una función muy importante en el suelo, mejorando propiedades como la estructura, turbación, capacidad de retención de agua, degradación de la materia orgánica, entre otros, estos contribuyen a la fertilidad del suelo y, por ende, al crecimiento del vegetal (Domínguez et al, 2010).

\section{Métodos y materiales}

El presente estudio se desarrolló en las plantaciones de Pinus tecunumanii de la Estación Experimental Agropecuaria Satipo, ubicado en el distrito Río Negro de la provincia Satipo.

La investigación es de tipo descriptivo - correlacional, se utilizó el diseño de muestreo estratificado, los estratos 
fueron definidos por las gradientes de crecimiento de las plantas. La población estuvo representada por la plantación de Pinus tecunumanii de la Estación Experimental Agropecuaria Satipo, de 5 años de instalada, la muestra fue de 9 parcelas de $9 \times 12 \mathrm{~m}$, en plantas y calicatas en suelo. La unidad de muestreo, fueron las plantas de Pinus tecunumanii, los horizontes A de cada parcela para el suelo. Se efectuó el reconocimiento de la plantación de Pinus tecunumanii, para delimitar las parcelas a evaluar mediante la identificación de plantas con limitado, regular y buen crecimiento. La selección de estratos de crecimiento se realizó por agrupación de plantas que tenían porte alto, medio y bajo de crecimiento, en las que se delimitó 9 parcelas de $108 \mathrm{~m}^{2}$, encontrándose 12 plantas de Pinus tecunumani por parcela, las que se evaluó como muestra.

Se tomaron datos de diámetro a $30 \mathrm{~cm}$ del suelo y altura total de las plantas en cada estrato y parcelas delimitadas. En cada parcela se realizaron calicatas de $80 \mathrm{~cm}$ de ancho por $150 \mathrm{~cm}$ de largo y $120 \mathrm{~cm}$ de profundidad, de donde se extrajeron las muestras para evaluar las propiedades del suelo.

Se evaluó el potencial hidrógeno, con un potenciómetro digital; el contenido de materia orgánica, con el método Walkley y Black; el fósforo, se extrajo con la solución Bray II; el potasio, se determinó por turbidimetria; el calcio y magnesio, por titulación con EDTA; la acidez cambiable, se extrajo con cloruro de potasio al uno normal y; la capacidad de intercambio catiónico efectivo, mediante suma de cationes cambiables. La textura, se evaluó mediante el método de Bouyucos; la densidad, aparente real y; porosidad, analisada por el método de la probeta.
Para evaluar la infiltración, se construyó cilindros de infiltración con baldes de plástico y tubos de PVC de tres pulgadas, cortados a $40 \mathrm{~cm}$ de alto y se colocó una cinta métrica en el interior del cilindro, durante la evaluación se colocaron en forma concéntrica los cilindros y se llenó los cilindros con agua, en un tiempo cero y se controló la velocidad de infiltración cada cinco minutos hasta los treinta minutos, momento que sirvió para calcular la infiltración. La profundidad efectiva, se evaluó midiendo el crecimiento de raíces en los horizontes de las calicatas.

Para comparar los estratos de crecimiento de Pinus tecunumanii y las características de los suelos, se realizó el análisis de varianza y la prueba de comparación de promedios Tukey, al $95 \%$ de confianza. Para determinar la relación entre el crecimiento de Pinus tecunumanii y, para las características de los suelos, se realizó el análisis de varianza de la regresión y el cálculo del coeficiente de determinación.

Para la evaluación de lombrices, se abre hoyos de 50 x $50 \mathrm{~cm}$ con una profundidad de $20 \mathrm{~cm}$, en los que se removió el suelo y se extrajo sobre un costal, separándose las lombrices para luego ser contadas.

\section{Resultados}

Al comparar las características fenotípicas de las plantas de Pinus tecunumani, se observa que existe diferencia de altura y diámetro de plantas (valor $\mathrm{p}<0,05$ ) entre los estratos evaluados. Siendo la plantación de 5 años de edad, se encontró una variada velocidad de crecimiento de las plantas de Pinus tecunumani, la cual se atribuye a la variada condición de los suelos. Esto se muestra en la Tabla 1.

\section{Tabla 1}

Características fenotípicas de las plantas de Pinus tecunumani

\begin{tabular}{lcccccc}
\hline \multicolumn{1}{c}{ Variable } & Fuente & $\begin{array}{c}\text { Suma de } \\
\text { cuadrados }\end{array}$ & $\begin{array}{c}\text { Grados de } \\
\text { libertad }\end{array}$ & $\begin{array}{c}\text { Cuadrado } \\
\text { medio }\end{array}$ & Razón - F & Valor - P \\
\hline \multirow{3}{*}{ Altura } & Entre grupos & 39,4538 & 2 & 19,72690 & 14,31 & 0,0052 \\
& Intra grupos & 8,2702 & 6 & 1,37837 & & \\
\hline \multirow{2}{*}{ Diámetro } & Total & 47,724 & 8 & & & \\
& Entre grupos & 55,9288 & 2 & 27,96440 & 5,51 & 0,0437 \\
& Intra grupos & 30,4283 & 6 & 5,07138 & & \\
\hline
\end{tabular}

Al comparar las medias de la altura y diámetro de Pinus tecunumani, se observa que las plantas del estrato bajo tiene una altura promedio de 2,68 $\mathrm{m}$ y las plantas del estrato medio tienen 4,61 m, las que no presentan diferencia estadística entre ellas, las plantas del estrato alto tienen una altura de 7,76 $\mathrm{m}$ y son estadísticamente superiores a las plantas de los estratos medio y bajo. La velocidad de crecimiento estimada $(0,54,0,92$ y 1,55 $\mathrm{m} / \mathrm{año}$ ) para cada estrato es inferior a lo reportado por
García et al. (1999), quienes indican que P. tecunumanii presenta tasas de crecimiento en altura de 2,44 m /año.

Las plantas del estrato bajo, tiene un diámetro promedio de 3,31 centímetros y las plantas del estrato medio, tienen $6,70 \mathrm{~cm}$, las que no presentan diferencia estadística entre ellas; las plantas del estrato alto, tienen un diámetro de $9,76 \mathrm{~cm}$ y son estadísticamente superiores a las plantas del estratos bajo. Esto se observa en la Tabla 2. 
Tabla 2

Comparación de medias de altura y diámetro de Pinus tecunumani

\begin{tabular}{cccc}
\hline Variable & Estratos & Media & $\begin{array}{c}\text { Grupos } \\
\text { homogéneos }\end{array}$ \\
\hline Altura $(\mathrm{m})$ & Bajo & 2,68333 & $\mathrm{~A}$ \\
ALS $\left(\mathrm{T}_{0,05}\right)=2,94127$ & Medio & 4,61333 & $\mathrm{~A}$ \\
& Alto & 7,76333 & $\mathrm{~B}$ \\
\hline \multirow{2}{*}{ Altura $(\mathrm{m})$} & Bajo & 3,31000 & $\mathrm{~A}$ \\
ALS $\left(\mathrm{T}_{0,05}\right)=5,64176$ & Medio & 6,70000 & $\mathrm{~A} \mathrm{~B}$ \\
& Alto & 9,40333 & $\mathrm{~B}$ \\
\hline
\end{tabular}

Por otra parte, al comparar las características químicas de los suelos en los estratos de Pinus tecunumanii, se encuentra que existe diferencia en el potencial hidrógeno y en el contenido de materia orgánica (Valor-P $<0,05)$ entre los estratos evaluados; mientras que, en el contenido de fósforo, potasio, calcio, magnesio, acidez cambiable y la capacidad de intercambio catiónico efectivo entre los estratos de Pinus tecunumanii, no muestran diferencia estadística. Esto se puede observar en la Tabla 3.

\section{Tabla 3}

Características químicas de los suelos en los estratos del Pinus tecunumani

\begin{tabular}{|c|c|c|c|c|c|c|}
\hline Variable & Fuente & Suma de cuadrados & $\begin{array}{c}\text { Grados de } \\
\text { libertad }\end{array}$ & $\begin{array}{c}\text { Cuadrado } \\
\text { medio }\end{array}$ & Razón - F & Valor - P \\
\hline \multirow{3}{*}{$\mathrm{pH}$} & Entre grupos & 0,5606 & 2 & 0,280300 & 13,20 & 0,0063 \\
\hline & Intra grupos & 0,1274 & 6 & 0,021233 & & \\
\hline & Total & 0,6880 & 8 & & & \\
\hline \multirow{3}{*}{$\begin{array}{l}\text { Materia } \\
\text { orgánica }\end{array}$} & Entre grupos & 6,72996 & 2 & 3,36498 & 14,40 & 0,0051 \\
\hline & Intra grupos & 1,40207 & 6 & 0,233678 & & \\
\hline & Total & 8,13202 & & & & \\
\hline \multirow{3}{*}{ Fósforo } & Entre grupos & 0,0282889 & 2 & 0,0291444 & 0,04 & 0,9613 \\
\hline & Intra grupos & 4,39573 & 6 & 0,732622 & & \\
\hline & Total & 4,45402 & & & & \\
\hline \multirow{3}{*}{$\begin{array}{c}\text { Calcio } \\
\text { cambiable }\end{array}$} & Entre grupos & 0,842222 & 2 & 0,421111 & 0,46 & 0,6521 \\
\hline & Intra grupos & 5,49833 & 6 & 0,916389 & & \\
\hline & Total & 6,34056 & 8 & & & \\
\hline \multirow{3}{*}{$\begin{array}{l}\text { Magnesio } \\
\text { cambiable }\end{array}$} & Entre grupos & 1,92389 & 2 & 0,961944 & 1,27 & 0,3478 \\
\hline & Intra grupos & 4,56 & 6 & 0,76 & & \\
\hline & Total & 6,48389 & & & & \\
\hline \multirow{3}{*}{$\begin{array}{c}\text { Potasio } \\
\text { cambiable }\end{array}$} & Entre grupos & 0,0008 & 2 & 0,0004 & 0,05 & 0,9557 \\
\hline & Intra grupos & 0,0526 & 6 & 0,00876667 & & \\
\hline & Total & 0,0534 & 8 & & & \\
\hline \multirow{3}{*}{$\begin{array}{c}\text { Acidez } \\
\text { cambiable }\end{array}$} & Entre grupos & 0,00442222 & 2 & 0,00221111 & 0,60 & 0,5807 \\
\hline & Intra grupos & 0,0222667 & 6 & 0,00371111 & & \\
\hline & Total & 0,0266889 & 8 & & & \\
\hline \multirow{3}{*}{$\mathrm{ClCe}$} & Entre grupos & 4,31629 & 2 & 2,15814 & 2,35 & 0,1760 \\
\hline & Intra grupos & 5,50147 & 6 & 0,916911 & & \\
\hline & Total & 9,81776 & 8 & & & \\
\hline
\end{tabular}

Al comparar las características químicas de los suelos en los estratos del Pinus tecunumanii, se encuentra que existe diferencia en espesor del horizonte A, profundidad efectiva, infiltración y cantidad de lombrices (Valor-P $<0,05$ ) entre los estratos evaluados, mientras que en el contenido de arena, arcilla, limo, densidad aparente densidad real y porosidad entre los estratos de Pinus tecunumanii, no existe diferencia. Esto se muestra en la Tabla 4. 
Tabla 4

Características fisicas y biológicas de los suelos en los estratos de Pinus tecunumani

\begin{tabular}{|c|c|c|c|c|c|c|}
\hline Variable & Fuente & Suma de cuadrados & $\begin{array}{c}\text { Grados de } \\
\text { libertad }\end{array}$ & $\begin{array}{l}\text { Cuadrado } \\
\text { medio }\end{array}$ & Razón - F & Valor - P \\
\hline \multirow{3}{*}{ Arena } & Entre grupos & 17,5788 & 2 & 8,78941 & 0,68 & 0,5426 \\
\hline & Intra grupos & 777652 & 6 & 12,9609 & & \\
\hline & Total & 95,344 & 8 & & & \\
\hline \multirow{3}{*}{ Arcilla } & Entre grupos & 35,5022 & 2 & 17,7511 & 0,85 & 0,4722 \\
\hline & Intra grupos & 124,924 & 6 & 20,8207 & & \\
\hline & Total & 160,427 & 8 & & & \\
\hline \multirow{3}{*}{ Limo } & Entre grupos & 18,0804 & 2 & 9,04018 & 0,42 & 0,6772 \\
\hline & Intra grupos & 130,321 & 6 & 21,7202 & & \\
\hline & Total & 148,401 & 8 & & & \\
\hline \multirow{3}{*}{$\begin{array}{l}\text { Densidad } \\
\text { aparente }\end{array}$} & Entre grupos & 0,00548889 & 2 & 0,00274444 & 0,60 & 0,5810 \\
\hline & Intra grupos & 0,0276667 & 6 & 0,00461111 & & \\
\hline & Total & 0,0331556 & 8 & & & \\
\hline \multirow{3}{*}{$\begin{array}{l}\text { Densidad } \\
\text { real }\end{array}$} & Entre grupos & 0,0358222 & 2 & 0,0179111 & 1,05 & 0,4056 \\
\hline & Intra grupos & 0,102067 & 6 & 0,170111 & & \\
\hline & Total & 0,137889 & 8 & & & \\
\hline \multirow{3}{*}{ Porosidad } & Entre grupos & 7,59887 & 2 & 3,79943 & 0,29 & 0,7601 \\
\hline & Intra grupos & 79,3841 & 6 & 13,2307 & & \\
\hline & Total & 86,983 & 8 & & & \\
\hline \multirow{3}{*}{$\begin{array}{l}\text { Espesor de } \\
\text { horizonte A }\end{array}$} & Entre grupos & 90,0956 & 2 & 45,0478 & 9,83 & 0,0128 \\
\hline & Intra grupos & 27,4933 & 6 & 4,58222 & & \\
\hline & Total & 117,589 & 8 & & & \\
\hline \multirow{3}{*}{$\begin{array}{l}\text { Profundidad } \\
\text { efectiva }\end{array}$} & Entre grupos & 1295,23 & 2 & 647,613 & 8,17 & 0,0194 \\
\hline & Intra grupos & 475,853 & 6 & 79,3089 & & \\
\hline & Total & 1771,08 & 8 & & & \\
\hline \multirow{3}{*}{ Infiltración } & Entre grupos & 1570,67 & 2 & 785,333 & 5,91 & 0,0382 \\
\hline & Intra grupos & 797,333 & 6 & 132,889 & & \\
\hline & Total & 2368,0 & 8 & & & \\
\hline \multirow{3}{*}{ Lombrices } & Entre grupos & 1606,89 & 2 & 803,444 & 9,62 & 0,0134 \\
\hline & Intra grupos & 501,333 & 6 & 83,5556 & & \\
\hline & Total & 2108,22 & 8 & & & \\
\hline
\end{tabular}

La cantidad de arcilla varía de 29,80 a $39,10 \%$, la cantidad de arcilla varía de 8,90 a $24,20 \%$, la cantidad de limo varía de 45,00 a 58,00\%, la clase textural a la que pertenecen son francos y francos limosos, las que son consideradas como suelos de textura media.

La densidad aparente varía de 0,95 a $1,14 \mathrm{t} / \mathrm{m}^{3}$, la porosidad varía de 50,83 a 60,16\%, y corresponden a suelos apropiados para el crecimiento de las plantas, la cual se demuestra con la profundidad media del crecimiento de raíces. La densidad real varía de 2,12 a $2,55 \mathrm{t} / \mathrm{m}^{3}$.

Al comparar las medias del potencial hidrógeno $(\mathrm{pH})$ de los suelos en los estratos de Pinus tecunumani, se observa que los suelos del estrato bajo tiene un $\mathrm{pH}$ de 4,70 y es superior a los suelos del estrato medio que tiene un $\mathrm{pH}$ de 4,09; esos valores, son considerados como extremadamente ácidos y muy fuertemente ácidos. Ello se muestra en la Tabla 5. 
Tabla 5

Comparación de medias de altura

y diámetro de Pinus tecunumani

\begin{tabular}{|c|c|c|c|}
\hline Variable & Estratos & Media & $\begin{array}{l}\text { Grupos ho- } \\
\text { mogéneos }\end{array}$ \\
\hline & Medio & 4,09333 & A \\
\hline \multirow{2}{*}{$\begin{array}{c}\mathrm{pH} \\
\operatorname{ALS}\left(\mathrm{T}_{0,05}\right)=0,365057\end{array}$} & Alto & 4,36333 & A B \\
\hline & Bajo & 4,70333 & B \\
\hline \multirow{3}{*}{$\begin{array}{c}\text { Materia orgánica } \\
\operatorname{ALS}\left(\mathrm{T}_{0,05}\right)=1,21105\end{array}$} & Bajo & 3,39000 & A \\
\hline & Medio & 3,71000 & A \\
\hline & Alto & 5,36333 & B \\
\hline \multirow{3}{*}{$\begin{array}{l}\text { Espesor de horizonte A } \\
\operatorname{ALS}\left(\mathrm{T}_{0,05}\right)=5,36278\end{array}$} & Medio & 14,1333 & A \\
\hline & Bajo & 15,4333 & A \\
\hline & Alto & 21,4000 & B \\
\hline \multirow{3}{*}{$\begin{array}{l}\text { Profundidad efectiva } \\
\operatorname{ALS}\left(\mathrm{T}_{0,05}\right)=22,3107\end{array}$} & Bajo & 53,3333 & A \\
\hline & Medio & 59,4000 & A B \\
\hline & Alto & 81,2667 & B \\
\hline \multirow{3}{*}{$\begin{array}{c}\text { Infiltración } \\
\operatorname{ALS}\left(\mathrm{T}_{0,05}\right)=28,8799\end{array}$} & Bajo & 22,0000 & A \\
\hline & Medio & 30,6667 & A B \\
\hline & Alto & 53,3333 & B \\
\hline \multirow{3}{*}{$\begin{array}{c}\text { Lombrices } \\
\operatorname{ALS}\left(\mathrm{T}_{0,05}\right)=22,9002\end{array}$} & Bajo & 10,0000 & A \\
\hline & Medio & 18,6667 & A \\
\hline & Alto & 41,6667 & B \\
\hline
\end{tabular}

El contenido de materia orgánica de los suelos en los estratos bajo y medio es de 3,39 y 3,71 kg por metro cuadrado del horizonte A y son significativamente inferiores a la materia orgánica de los suelos del estrato alto que tienen $5,36 \mathrm{~kg}$ por metro cuadrado, si bien el contenido de materia orgánica expresados en porcentaje varían de 1,9 a 2,74 \%, no presentan diferencia estadística, pero si lo expresamos en kilos por metro cuadrado del horizonte A, se deja notar su diferencia, así como su efecto en el crecimiento de las plantas.

El espesor del horizonte A, los suelos en los estratos bajo y medio es de 14,13 y 15,43 cm de espesor y son significativamente inferiores espesor de los suelos del estrato alto que presentan $21,40 \mathrm{~cm}$.

La profundidad efectiva en los suelos son considerados como moderadamente profundos, en el estrato bajo es de $53,33 \mathrm{~cm}$, en el estrato medio es de 59,40 cm, mientras que en el estrato alto es $81,27 \mathrm{~cm}$ y es significativamente superior al estrato bajo.

La infiltración en los suelos en el estrato bajo es de $22,00 \mathrm{ml}$ por hora, en el estrato medio es de $30,67 \mathrm{ml}$ por hora, mientras que en el estrato alto es $53,33 \mathrm{ml}$ por hora centímetros y es significativamente superior al estrato bajo. La cantidad de lombrices en los suelos en los estratos bajo y medio es de 10,00 y 18,67 lombrices en promedio y son significativamente inferiores a la cantidad de lombrices de los suelos del estrato alto que tienen 41,67 lombrices (en 0,25 $\mathrm{m}^{2}$ y a 0,2 $\mathrm{m}$ de profundidad).

El análisis de regresión entre las características del suelo, muestra que el contenido de materia orgánica en el horizonte A, la profundidad efectiva, la velocidad de infiltración y la cantidad de lombrices, muestran una relación estadísticamente significativa, esto revela que al cambiar estas características, influyen en el crecimiento de Pinus tecunumanii, a los 5 años de haber establecido la plantación. El análisis se muestra en la Tabla 6.

\section{Tabla 6}

Análisis de regresión entre las características del suelo y el crecimiento del Pinus tecunumani

\begin{tabular}{|c|c|c|c|c|c|c|}
\hline Variable & Fuente & $\begin{array}{c}\text { Suma de } \\
\text { cuadrados }\end{array}$ & $\begin{array}{c}\text { Grados de } \\
\text { libertad }\end{array}$ & $\begin{array}{l}\text { Cuadrado } \\
\text { medio }\end{array}$ & Razón - F & Valor - P \\
\hline \multirow{3}{*}{$\begin{array}{l}X=\text { Materia orgánica } \\
Y=\text { Altura de planta } \\
r=0,7379 ; r^{2}=0,5446\end{array}$} & Modelo lineal & 25,9892 & 1 & 25,9892 & 8,37 & 0,0232 \\
\hline & Residuo & 21,7348 & 7 & 3,1050 & & \\
\hline & Total & 47,7240 & 8 & & & \\
\hline \multirow{3}{*}{$\begin{array}{l}X=\text { Profund. efectiva } \\
Y=\text { Altura de planta } \\
r=0,6852 ; r^{2}=0,4695\end{array}$} & Modelo multip. & 0,96247 & 1 & 0,9625 & 6,19 & 0,0417 \\
\hline & Residuo & 1,08762 & 7 & 0,1554 & & \\
\hline & Total & 2,05009 & 8 & & & \\
\hline \multirow{3}{*}{$\begin{array}{c}\mathrm{X}=\text { Infiltración } \\
\mathrm{Y}=\text { Altura de planta } \\
\mathrm{r}=0,7039 ; \mathrm{r}^{2}=0,4955\end{array}$} & Modelo lineal & 23,648 & 1 & 23,6480 & 6,88 & 0,0343 \\
\hline & Residuo & 24,076 & 7 & 3,4394 & & \\
\hline & Total & 47,724 & 8 & & & \\
\hline \multirow{3}{*}{$\begin{array}{c}\mathrm{X}=\text { Lombrices } \\
\mathrm{Y}=\text { Altura de planta } \\
\mathrm{r}=0,8738 ; \mathrm{r}^{2}=0,7636\end{array}$} & Modelo lineal & 36,4424 & 1 & 36,4424 & 22,61 & 0,0021 \\
\hline & Residuo & 11,2816 & 7 & 1,6116 & & \\
\hline & Total & 47,7240 & 8 & & & \\
\hline
\end{tabular}


Relación entre las características del suelo y las características de las plantas de Pinus tecunumanii

Al relacionar la altura de Pinus Tucunumanii y el contenido de materia orgánica del horizonte $\mathrm{A}$, se aprecia que al aumentar el contenido de materia orgánica se incrementa la altura de plantas, el coeficiente de determinación (r2), indica que el 50,46\% de la variación de la altura de Pinus Tucunumanii depende de la cantidad de materia orgánica del horizonte A. Esta relación de dependencia se debe a que la materia orgánica por acción de los macro y micro organismos provee de nutrientes para el crecimiento de las plantas; así mismo, favorece la retención de agua disponible para las plantas y mejora la estructura del suelo. Esto se puede observar en la Figura 1.

Figura 1

Relación entre la altura de Pinus Tucunumanii y

el contenido de materia orgánica del horizonte $A$

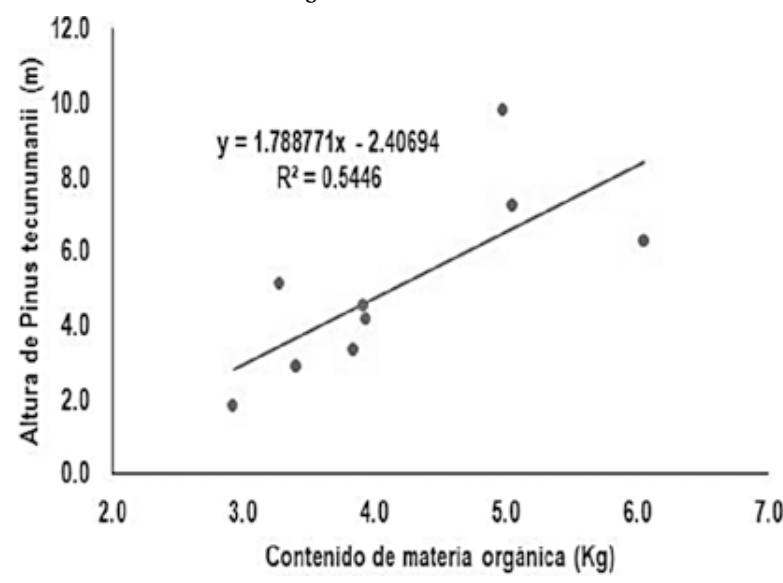

Al relacionar la altura de Pinus Tucunumanii y la profundidad efectiva, se aprecia que al aumentar la profundidad efectiva se incrementa la altura de plantas, el coeficiente de determinación (r2), indica que el 46,95\% de la variación de la altura de Pinus Tucunumanii depende de la profundidad efectiva. Esta relación de dependencia se debe al mayor volumen de suelo que las raíces pueden aprovechar para su nutrición cuando mayor es la profundidad de crecimiento de las raíces en el suelo. La relación se muestra en la Figura 2.

\section{Figura 2}

Relación entre la altura de Pinus Tucunumanii

y la profundidad efectiva del suelo

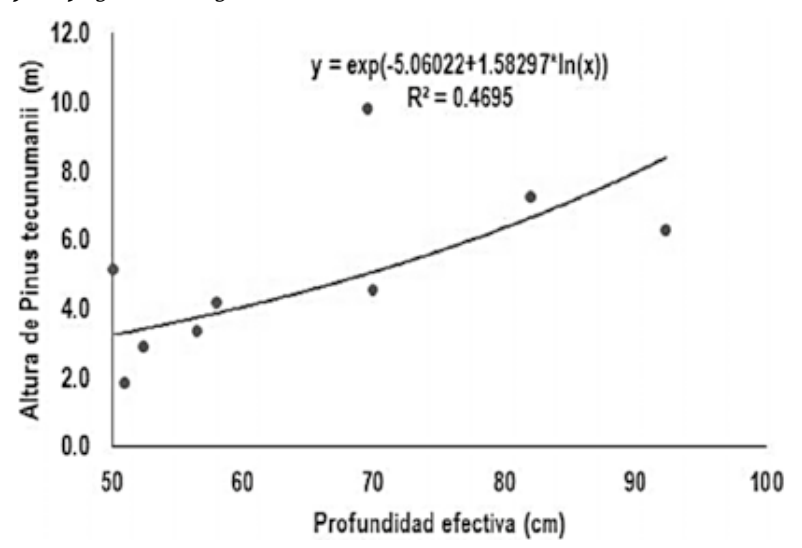

Al relacionar la altura de Pinus Tucunumanii y la velocidad de infiltración, se aprecia que al aumentar la velocidad de infiltración se incrementa la altura de plantas, el coeficiente de determinación (r2), indica que el 49,55\% de la variación de la altura de Pinus Tucunumanii depende de la velocidad de infiltración. Esta relación de dependencia, se debe a que al haber mayor infiltración, el suelo tiene mayor cantidad de agua disponible para las plantas, esta condición de humedad favorece la actividad de los organismos del suelo encargados de solubilizar nutrientes para las plantas. La relación se muestra en la Figura 3.

\section{Figura 3}

Relación entre la altura de Pinus Tucunumanii y la velocidad de infiltración

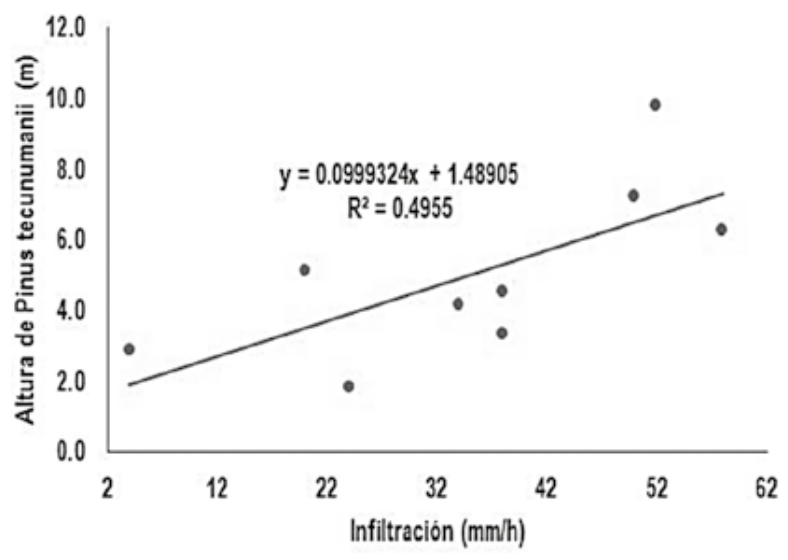

Al relacionar la altura de Pinus Tucunumanii y la cantidad de lombrices, se aprecia que al aumentar la cantidad de lombrices se incrementa la altura de plantas, el coeficiente de determinación (r2), indica que el 76,36\% de la variación de la altura de Pinus Tucunumanii depende de la cantidad de lombrices. El incremento de lombrices del suelo está relacionado con la materia orgánica, la cual sirve de alimento para las lombrices, las que a su vez enriquecen el suelo con microorganismos que proliferan en su tracto digestivo y se encargan de liberar nutrientes para las plantas. Esto se observa en la Figura 4.

\section{Figura 4}

Relación entre la altura de Pinus Tucunumanii y la cantidad de lombrices

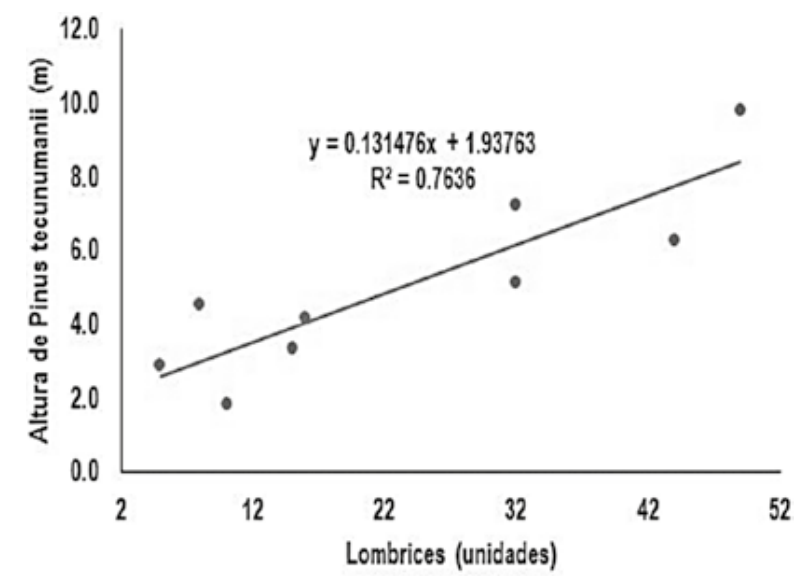




\section{Discusión}

La velocidad de crecimiento estimada de Pinus tecunumani $(0,54,0,92$ y 1,55 m/año) para cada estrato y es inferior a lo reportado por García et al. (1999), quienes indican que $P$. tecunumanii presenta tasas de crecimiento en altura de 2,44 m /año. El estrato bajo, con menor crecimiento de la plantas de Pinus tecunumani, está en la parte alta de la plantación, tal como lo reporta Domínguez et al. (2017), quienes indican que el crecimiento en altura y diámetro de Pinus greggii Engelm, fueron significativamente mayores en sitios inferiores del terreno, y coincide con Ramos, Castro y Sánchez (2015), quienes resaltan que la pendiente influye en la acumulación o lavado de nutrientes y materia orgánica.

Características del suelo, como la materia orgánica, velocidad de infiltración, profundidad de efectiva (crecimiento de raíces) y cantidad de lombrices, son diferentes en los suelos de la plantación de Pinus tecunumani. El análisis de la materia orgánica, reporta un menor contenido en suelos del estrato bajo (con plantas de menor crecimiento) que se encontraban en la parte superior de la parcela, tal como los sugiere Foth (1990) los nutrientes y la materia orgánica se escurren hacia la parte baja del terreno. Este efecto permite que al variar la materia orgánica, también varié la cantidad de lombrices, debido principalmente a que ellas se alimentan de la materia orgánica.

La mayor velocidad de infiltración, también se da en suelos que tienen mayor cantidad de materia orgánica, esta característica permite que las plantas crezcan con mayor rapidez en suelos, que tienen mayor permeabilidad, las que también permiten una mayor disponibilidad de agua y nutrientes para las plantas.

Martínez et al. (2013) manifiestan que en sitios con diferente productividad (alta, media y baja), identificaron diferencias significativas en la profundidad de suelo. Esta información coincide con los resultados obtenidos, ya que las plantas con mayor crecimiento se encuentran en suelos que tienen mayor profundidad efectiva. Los resultados también revelan una relación con el desarrollo del perfil de los suelos, debido a que los suelos con plantas más altas, presentaban horizonte A, B y C, mientras que los suelos con plantas de menor crecimiento, presentaban horizontes A y $\mathrm{C}$, según Eguiluz \& Perry, (s.f.), los suelos profundos, permiten un mayor crecimiento de las plantas.

Las lombrices, según Domínguez (2004), estimula la actividad microbiana e incrementa la mineralización de los nutrientes y, además, de mejora la disponibilidad de nutrientes; por lo que, se puede afirmar que mejora la fertilidad de los suelos. Este efecto está relacionado al mayor crecimiento de plantas en suelos que tienen mayor cantidad de lombrices.
El contenido de fósforo, potasio, calcio y magnesio son bajos y medios, y se consideran como suelos de baja fertilidad. Y, según Eguiluz y Perry (s.f.), Pinus tecunumani prospera mejor en suelos moderadamente fértiles, la baja fertilidad de los suelos de la plantación de Pinus tecunumani condiciona la menor velocidad de crecimiento, y según García et al. (1999), las plantan crecen de 63,52 a 22,13\% de lo esperado. El crecimiento también estaría limitado por las condiciones de acidez de los suelos, los resultados indican que los suelos de la parcela son extremadamente ácidos y muy fuertemente ácido, ya que, según Eguiluz \& Perry (s.f.), la distribución de Pinus tecunumani se da en suelos ligeramente ácidos a neutros, aunque Quispe (s.f.) menciona que prefiere suelos ácidos. Al relacionar las propiedades de los suelos del horizonte A, se encontró una fuerte relación entre el contenido de materia orgánica, la profundidad efectiva, la cantidad de lombrices y el crecimiento de Pinus tecunumani. Al incrementarse la materia orgánica del suelo se incrementa el crecimiento de las plantas, así lo sugieren Pascual, Izquierdo y Venegas (s.f.), quienes dicen que la materia orgánica, mejora la estructura, previene la erosión y mejora la retención de nutrientes; por su parte Arancel (2012), encontró que la producción del café está relacionada en un $28,70 \%$ con la cantidad de materia orgánica.

También, se encontró que al tener mayor profundidad efectiva, las plantas crecen mejor, ya que al tener mayor volumen de suelo en contacto con las raíces, las plantas tienen mayor disponibilidad de nutrientes, así Griffiths et al. (2009) encontraron que existe relación entre el crecimiento de coníferas y la profundidad del suelo. Además, suelos con buen drenaje permiten que las plantas, además de tener agua, también tengan oxígeno, lo que permite el establecimiento y crecimiento de las plantas; en ese sentido, Eguiluz \& Perry, (s.f.) afirman que la distribución de Pinus tecunumani se da en suelos bien drenados. Además Vallejo y Quintero (2013), indican que una buena porosidad, que facilita la aireación y el drenaje, se dan en suelos que no presentan problemas de compactación.

Esto lo reafirma Ramírez (1997), al indicar que las características físicas de los suelos son responsables del crecimiento de las plantas. Y que la distribución de $P_{i-}$ nus tecunumani se da en suelos bien drenados (Eguiluz \& Perry, s.f.).

La distribución de lombrices está fuertemente relacionada al crecimiento de las planta, esta relación no indica que las lombrices influyen directamente en la producción o crecimiento de las plantas, sino que lo hacen a través de la mejor infiltración de agua en el suelo, debido a las galerías que hacen las lombrices al desplazarse en el perfil del suelo. Mientras que, Domínguez (2004) indica que las lombrices estimulan la actividad microbiana 
e incrementa la mineralización de los nutrientes y que, además de mejorar la disponibilidad de nutrientes, mejora las propiedades físicas. Por su parte, Arancel (2012) encontró que la producción del café está relacionada en un 65,33\% con la cantidad de lombrices.

En términos generales, Bonilla (1971) resalta que los factores edafológicos influyen en el crecimiento de los árboles; analizar la relación de cada una de esos factores, permite mejorar la producción de madera y la eficiencia del uso del suelo. Así mismo, Ramírez (1997) indica que la interacción de las características químicas, físicas y biológicas permiten el óptimo crecimiento de las plantas.

\section{Conclusiones}

- Se encontró una variada velocidad de crecimiento de las plantas de Pinus tecunumani, debido a la variada condición de los suelos. las plantas del estrato bajo tiene una altura promedio de 2,68 $\mathrm{m}$, las plantas del estrato medio tienen 4,61 m y las plantas del estrato alto tienen una altura de 7,76 m.

- Las características químicas de los suelos en los estratos de Pinus tecunumanii, se encuentra que existe dife-rencia en el potencial hidrógeno y en el contenido de materia orgánica (Valor- $\mathrm{P}<0,05$ ); mientras que, en el contenido de fósforo, potasio, calcio, magnesio, acidez cambiable y la capacidad de intercambio catiónico efectivo, no muestran diferencia estadística.

- El contenido de materia orgánica de los suelos en los estratos bajo y medio es de 3,39 y 3,71 kg por me-tro cuadrado del horizonte A y son significativamente inferiores a la materia orgánica de los suelos del estrato alto que tienen $5,36 \mathrm{~kg}$ por metro cuadrado.

- La cantidad de lombrices en los suelos en los estratos bajo y medio es de 10,00 y 18,67 lombrices en promedio y son significativamente inferiores a la cantidad de lombrices de los suelos del estrato alto que tienen 41,67 lombrices (en 0,25 m2 y a 0,2 m de profundidad).

\section{Referencias bibliográficas}

Alvarado, A. y Raigosa, J. (2007). Nutrición y fertilización forestal en regiones tropicales. Centros de Investigaciones Agronómicas. Universidad de Costa Rica San José, Costa Rica pp 83.

Arias, A. (2007). Suelos tropicales. Capitulo 4. Propiedades del suelo. Primera edición, EUNED, San José de Costa Rica. Pág. 63.

Aristisabal, M. (2003). Fisiología vegetal en Colombia. Ed. Artes gráficas Tisan ISBN 9583354171V.1P.306.
Bellote, A. \& Farreira, C. (1995). Nutrientes minerales y crecimiento de árboles abonados de Eucalyptus grandis en el Estado de São Paulo. Bosque (Valdivia), 16(1), 69- 75.

Cárdenas Alhuay, E. (2013). Efecto de hidrogel en el crecimiento inicial de Guazuma crinita Mart., Pinus tecunumanii (Eguiluz \& Perry) y Cedrela fissilis Vell.-distrito de Pichanaqui.

Domínguez, J.; Lazcano, C. \& Gómez-Brandón, M. (2010). Influencia del vermicompost en el crecimiento de las plantas: Aportes para la elaboración de un concepto objetivo. Acta Zoológica Mexicana, 26(SPE2), 359-371.

Domínguez-Calleros, P. A.; Rodríguez-Laguna, R.; Capulín-Grande, J.; Razo-Zárate, R. \& Díaz-Vásquez, M. A. (2017). Influencia de factores edáficos en el crecimiento de una plantación de Pinus greggii Engelm. en Santiago de Anaya, Hidalgo, México. Madera y bosques, 23(2), 145-154.

Eguiluz \& Perry. (s.f.) Pinus tecunumanii.PMD - Arboles de Centroamerica. Homepage, consultado el 02 de junio de 2019, disponible en www.arbolesdecentroamerica.info/.../es/.../198_111ec7368f256e09425eof0ec0d 8b78...

García, J. G.; Hernández, J. J.; Mata, J. J.; Galán, J. D.; Herrera, C. R. \& Upton, J. L. (1999). Variación en el patrón de crecimiento en altura de cuatro especies de Pinus en edades tempranas. Madera y Bosques, 5(2), 19-34.

González, J. (2014) Efecto del uso y ocupación en las propiedades físicas y químicas en un suelo del piedemonte llanero. Tesis de maestría. Bogotá, Colombia.

Grubb, P. J. (1995). Mineral nutrition and soil fertility rain forest. In : A.e. Lugo y C. Lowe (Eds. ) Tropical Forest: management an ecology. Springer- Berlaj, Ecologycal studies v. 112. New York, USA Pp. 308-330.

Harison, L. (2001). Fertilidad de suelos. Un artículo fundamental, Permacultura, México.

INTAGRI, S. C., Propiedades físicas del suelo y el crecimiento de las plantas. Disponible en: https://www.intagri. com/articulos/suelos/propiedades-fisicas-del-suelo-y-elcrecimiento-de-las-plantas.

Ivañez, J. (2008). Fertilidad física, química y biológica. Biología y Ecología del suelo. CSIC Universidad de Valencia España.

Jaramillo, D. (2002). Introducción a la ciencia del suelo. Universidad Nacional de Colombia Medellín. Pp. 331-334. 
Jeddi, K. \& Chaieb, M. (2010). Soil properties and plant community in different aged Pinus halepensis Mill. plantations in arid Mediterranean areas: The case of southern Tunisia. Land degradation \& development, 21(1), 32-39.

Juan De Dios, C. (2015). Efecto de sustratos comerciales en la germinación y crecimiento inicial de pinus oocarpa schiede ex schltdl. y Pinus tecunumanii F. Schwerdtf. ex Eguiluz \&J. P. Perry En Condiciones de vivero - San Ramón-Chanchamayo. Tesis.

Martínez, E.; Fuentes, J. P. \& Acevedo, E. (2008). Carbono orgánico y propiedades del suelo. Revista de la ciencia del suelo y nutrición vegetal, 8(1), 68-96.

Martínez-Salvador, M.; Valdez-Cepeda, R. D. \& Pompa García, M. (2013). Influencia de variables físicas en la productividad de Pinus arizonica y Pinus engelmannii en el sur de Chihuahua, México. Madera y bosques, 19(3), 35-49.

Minag. (2009). Decreto Supremo No 017-2009-Ag. Reglamento de clasificación de tierras por su capacidad de uso mayor.

Moya, R.; Arce, V.; Gonzàlez, E.; Olivares, C. \& Rios, V. (2010). Eefeito das propriedades físicas e quí- micas do solo em algumas propriedades da madeira de teca (Tectona grandis). Revista Árvore, 34(6), 1109- 1118. https://dx.doi.org/10.1590/S010067622010000600017

Osorio, N. (2008). Ciclaje de nutrientes en rodales de distintas edades, establecidas en el Caribe Seco Colombiano. Publicado por Nutrición forestal.

Quispe, A. (s.f.) Semillas de Pinus tecunumanii "Pino rojo" - Arborizaciones, disponible en arborizaciones. com/pluginfile.php/.../mod_folder/content/0/ Pinus\%20tecunumanii \%2...

Ramírez R. 1997. Propiedades físicas, químicas y biológicas de los suelos. Santa Fé de Bogotá, OC.

Silva, A. (1998). La materia orgánica del suelo. Montevideo: Facultad de Agronomía. 34 p.

Thiers, O.; Reyes, J.; Gerding, V. \& Schlatter, J. E. 2014. Suelos en ecosistemas forestales. In Donoso C, ME

Vallejo-Quintero, V. E. (2013). Importancia y utilidad de la evaluación de la calidad de suelos mediante el componente microbiano: experiencias en sistemas silvopastoriles. Colombia forestal, 16(1), 83-99. 